\title{
CoviDecode : Detection of COVID-19 from Chest X-Ray images
} Networks

\author{
Rishabh Raj
}

Student, Information Technology, Maharaja Agrasen Institute of Technology, New Delhi.

To Cite this Article

Rishabh Raj, "CoviDecode : Detection of COVID-19 from Chest X-Ray images using Convolutional Neural Networks", International Journal for Modern Trends in Science and Technology, 6(12): 436-439, 2020.

\section{Article Info}

Received on 16-November-2020, Revised on 09-December-2020, Accepted on 12-December-2020, Published on 18-December-2020.

\section{ABSTRACT}

Deep learning plays a major role from past years in image detection, spam reorganization, normal speech command, product recommendation and medical diagnosis. The detection of severe acute respiratory syndrome corona virus 2 (SARS CoV-2), which is responsible for corona virus disease 2019 (COVID-19), using chest $X$-ray images has life-saving importance for bothpatients and doctors. In addition, in countries that are unable to purchase laboratory kits for testing, this becomes even more vital. In this study, we aimed to present the use of deep learning for the high-accuracy detection of COVID-19 using chest X-ray images. Publicly available X-ray images were used in the experiments, which involved the training of deep learning and machine learning classifiers. Experiments were performed using convolutional neural networks and machine learning models. Images and statistical data were considered separately in the experiments to evaluate the performances of models, and eightfold cross-validation was used. A mean accuracy of 98.50\%. A convolutional neural network without pre-processing and with minimized layers is capable of detecting COVID- 19 in a limited number of, and in imbalanced, chest X-rayimages.

KEYWORDS:COVID-19, deep learning, convolutional neural networks, SARS CoV-2

\section{INTRODUCTION}

Corona viruses are a family of hundreds of viruses that can cause fever, respiratory problems, and sometimes gastrointestinal symptoms too. The 2019 novel corona virus is one of seven members of this family known to infect humans, and the third in the past threedecades to jump from animals to humans. Since emerging in China in December, this new corona virus has caused a global health emergency, sickening almost 200,000 people worldwide, and so far killing more than 9,000. As of March 19, about 10000 cases had been reported in the assumed to be 20 times this number. The basic reproduction number (BRN) is the expected number of cases directly generated by one case. A
BRN greater than one indicates that the outbreak is self-sustaining, while a BRN less than one indicates that the number of new cases decreases over time and eventually the outbreak will stop. Ideally, the BRN should be reduced in order to slow down an epidemic. The BRN in the first three phases was estimated to be $3.1,2.6$, and 1.9, respectively. In the Cell Discovery article, the BRN is assumed to have decreasedto 0.9 or 0.5 in phase IV, based on previous experience in SARS. According to an article in Science in 2003, the BRN of SARS decreased from 2.7 to 0.25 after the patients were isolated and the infection started being controlled. 
The better we can track the virus, the better we can fight it. By analyzing different parameters responsible for the outbreak of corona virus, we can take controlling measures in an accelerated way.

\section{DATASET}

The dataset used here is the real dataset. The images are gathered from Github and Kaggle and the images are of two types Normal chest X-Ray of people who are not affected and of those of people who are affected and are positive of COVID-19.

\section{LITERATURE SURVEY}

Literature survey is the most important step in any kind of research. Before start developing we need to study the previous papers of our domain which we are working and on the basis of study we can predict or generate the drawback and start working with the reference of previous papers. In this section, we briefly review the related work on Chest $\mathrm{X}$-Rays and the techniques used. There are many factors that have an impact on this model of predicting the disease. Discovering corona virus manually is a difficult task and generally not very accurate and also not very safe, hence this model is developed to help the doctors and health workers to identify the disease without getting in contact with the patient. Boran Sekeroglu and Ilker Ozsahin had proposed an advanced Covid virus prediction model using CNN. This system's aim was to make a model that works on 3 classes covid-19/Normal, Covid19/Pneumonia andCovid-19/Pneumonia/Normal.

This paper [1] performed on thirty-eight experiments were performed using convolutional neural networks, 10 experiments were performed using five machine learning models, and 14 experiments were performed using the state-ofthe-art pre-trained networks for transfer learning. Images and statistical data were considered separately in the experiments to evaluate the performances of models, and eightfold crossvalidation was used. A mean sensitivity of $93.84 \%$, mean specificity of $99.18 \%$, mean accuracy of $98.50 \%$, and mean receiver operating characteristics-area under the curve scores of $96.51 \%$ are achieved.

The paper[2] proposed by Pranav Rajpurkar * 1 Jeremy Irvin * 1 Kaylie Zhu 1 Brandon
Yang 1 Hershel Mehta 1 Tony Duan 1 Daisy Ding 1 Aarti Bagul 1 Robyn L. Ball 2 Curtis Langlotz 3 Katie Shpanskaya 3 Matthew P. Lungren 3 Andrew Y. Ng 1 developed an algorithm that can detect pneumonia from chest X-rays at a level exceeding practicing radiologists. Our algorithm, CheXNet, is a 121- layer convolutional neural network trained on ChestX-ray14, currently the largest publicly available chest Xray dataset, containing over 100,000 frontalview X-ray images with 14 diseases. Four practicing academic radiologists annotate a test set, on which we compare the performance of CheXNet to that of radiologists. We find that CheXNet exceeds average radiologist performance on the $\mathrm{F} 1$ metric. We extend CheXNet to detect all 14 diseases in ChestX-ray 14 and achieve state of the art results on all 14 diseases. They also show that a simple extension of our algorithm to detect multiplediseases outperforms previous state of the art onChestX-ray 14, the largest publicly available chest Xray dataset. With automation at the level of experts, we hope that this technology can improve healthcare delivery and increase access to medical imaging expertise in parts of the world where access to skilled radiologists is limited.

\section{MethodologY}

The methodology will go according to the following flowchart.

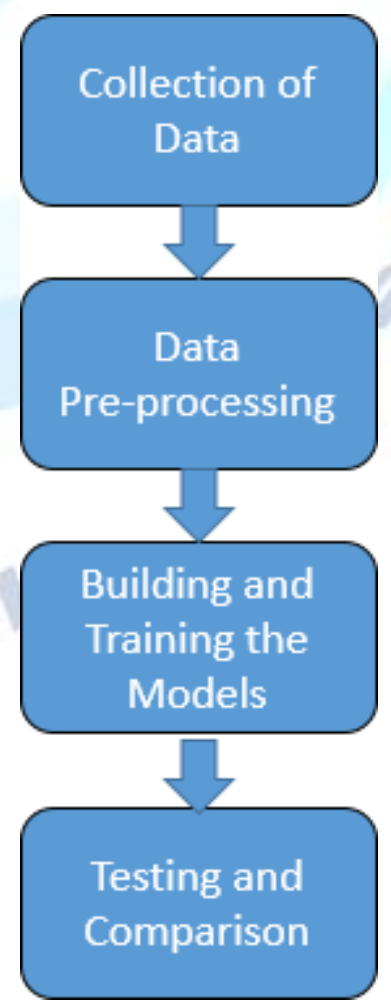

In this study, a deep learning model is proposed for the automatic diagnosis of COVID-19. The 
proposed model has an end-to-end architecture and it requires raw chest X-ray images to return the diagnosis. This model is trained with 200 chest $\mathrm{X}$-ray images, which are not in a regular form and were obtained hastily.. One of the most important disadvantages of chest radiography analyses is an inability to detect the early stages of COVID-19, as they do not have sufficient sensitivity in GGO detection However, well- trained deep learning models can focus on points that are not noticeable to the human eye, and may serve to reverse this perception.

\section{Material and methods}

\section{$X$ - ray imageDataset}

In this study, X-ray images obtained from two different sources were used for the diagnosis of COVID-19 using images from various open access sources. This database is constantly updated with images shared by researchers from different regions. At present, there are $200 \mathrm{X}$-ray images diagnosed with COVID-19 in the database.

The proposed CoviDecode model

The advent of deep learning technology has revolutionized artificial intelligence The word deep refers to the increase in the size of this network with the number of layers. The structure is named after convolution, a mathematical operator. A typical CNN structure has a convolution layer that extracts features from the input with the filters it applies, a pooling layer to reduce the size for computational performance, and a fully connected layer, which is a neural network. By combining one or more such layers, a CNN model is created, and its internal parameters are adjusted to accomplish a particular task, such as classification or object recognition.

This model contains convolutional layers along with other layers such as max pooling which helps to select the maximum element from the region of the feature map covered by the filter. Thus, the output after max-pooling layer would be a feature map containing the most prominent features of the previous feature map.

Along with Max Pooling layer padding layer is also added in order to make sure that the filter fits the input image perfectly. The layer we call as FC or fully connected layer, we flattened our matrix into vector and feed it into a fully connected layer like a neural network.

The activation function used here is ReLu . It stands for Rectified Linear Unit for a non-linear operation. The output is $\boldsymbol{f}(\boldsymbol{x})=\boldsymbol{m a x}(\boldsymbol{0}, \boldsymbol{x})$. ReLU's purpose is to introduce non-linearity in our ConvNet. Since, the real world data would want our
ConvNet to learn would be non-negative linear values.

\section{RESULTS}

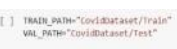

Fig-1 (Specifying path)
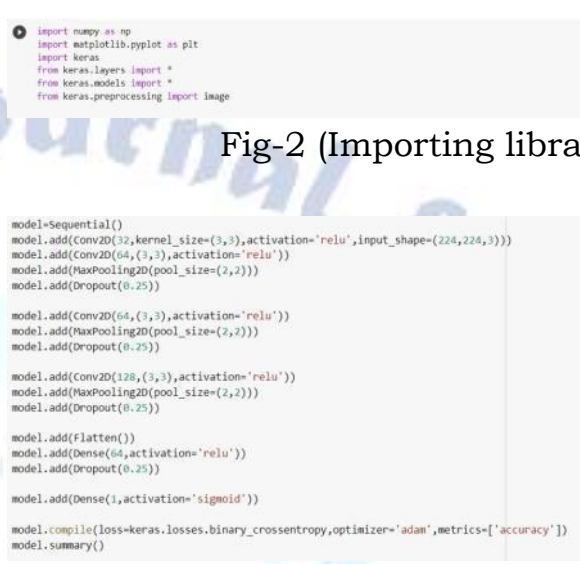

Fig-3.1 (CreatingModel)

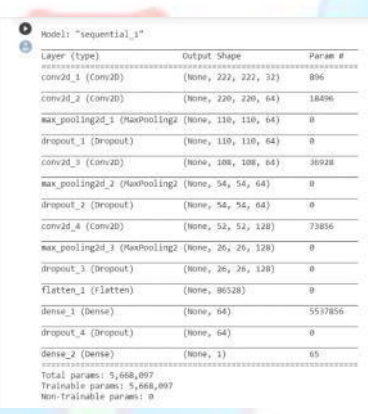

Fig-3.2 (CreatingModel)

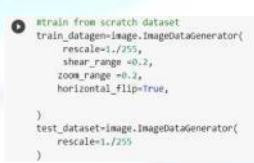

Fig-4.1 (TrainingModel)

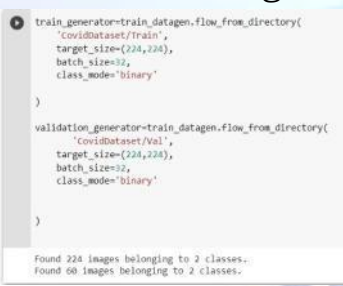

Fig-4.2 (TrainingModel)

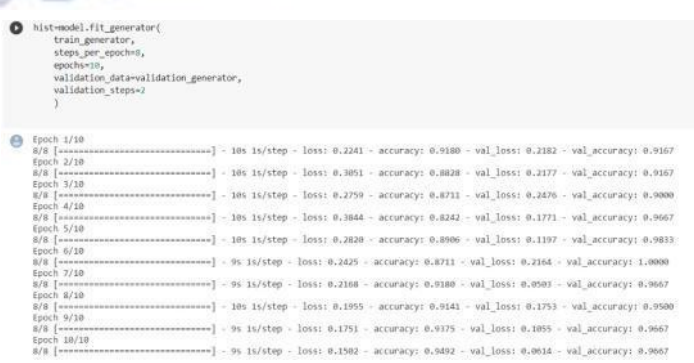

Fig-5 (DisplayingResult) 
Accuracy of almost $96 \%$ has been achieved with the given training data and almost $97 \%$ with the given validation data.

\section{CONCLUSION}

Detection of COVID-19 from chest X-ray images is of vital importance for both doctors and patients to decrease the diagnostic time and reduce financial costs. Artificial intelligence and deep learning are capable of recognizing images for the tasks taught. In this study, several experiments were performed for the high- accuracy detection of COVID-19 in chest X-ray images . Various groups-COVID-19/Normal and COVID-19/Positive- were considered for the classification. Different image dimensions, different network architectures, and machine learning models were implemented and evaluated using images and statistical data. When the number of images in the database and the detection time of COVID-19 are considered, it can be suggested that the considered architectures reduce the computational cost with high performance. The results showed that the convolutional neural network with minimized convolutional and fully connected layers is capable of detecting COVID-19 images within the two-class, COVID-19/Negative and COVID- 19/ Positive. Therefore, the use of AI-based automated high-accuracy technologies may provide valuable assistance to doctors in diagnosing COVID-19. Further studies, based on the results obtained in this study, would provide more information about the use of CNN architectures with COVID-19 chest $\mathrm{X}$-ray images and improve on the results of thisstudy.

\section{REFERENCES}

- Detection of COVID-19 from Chest X-Ray Images Using Convolutional Neural Networks Boran Sekeroglu and Ilker Ozsahin

- CheXNet: Radiologist-Level Pneumonia Detection on Chest X-Rays with Deep Learning Pranav Rajpurkar, Jeremy Irvin, Kaylie Zhu, Brandon Yang, Hershel Mehta, Tony Duan, Daisy Ding, Aarti Bagul, Robyn L. Ball, Curtis Langlotz, Katie Shpanskaya, Matthew P. Lungren, Andrew Y. Ng

- $\quad$ Rubin, G. D.; Ryerson, C. J.; Haramati, L. B.; et al. The Role of Chest Imaging in Patient Management during the COVID- 19 Pandemic: A Multinational Consensus Statement from the Fleischner Society [published online ahead of print, 2020 Apr7]. Chest. 2020, S0012-3692, 30673- 30675.doi:10.1016/j.chest.2020.04.003.

- Ozsahin, I.; Sekeroglu, B.; Mok, G. S. P. The Use of Back Propagation Neural Networks and 18F-Florbetapir PET for
Early Detection of Alzheimer'sDisease

Using Alzheimer's Disease Neuroimaging Initiative Database. PLoS One. 2019, 14, e0226577.

- Apostolopoulos, I. D.; Mpesiana, T. Covid-19: Automatic Detection from X- Ray Images Utilizing Transfer Learning with Convolutional Neural Networks. Phys. Eng. Sci. Med.2020, 43,635-640.

For Dataset: $\underline{\text { http://cb.1k/covid_19 }}$

OTHERS:

- https://codebasicshub.com/

- https://www.coursera.org/

- World Health OrganizationWHO Coronavirus Disease (COVID-19) Dashboard

- https://covid19.who.int

- https://blog.datawow.io/interns-explain-cnn-8a669d053f8 $\underline{\mathrm{b}}$

- en.wikipedia.org

- https://www.mathworks.com/discovery/ convolutional-neural-network.html

- https://adeshpande3.github.io/adeshpande3.github.io/A -Beginner's-Guide-To-Understanding-Convolutional-Neura 1-Networks/

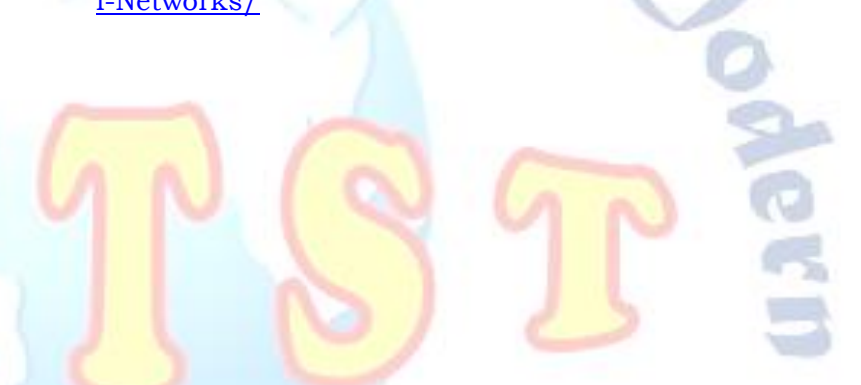

